\title{
Analgesic effects of naringenin in rats with spinal nerve ligation-induced neuropathic pain
}

\author{
CHUAN YIN HU ${ }^{1}$ and YUN-TAO ZHAO ${ }^{2}$ \\ ${ }^{1}$ Department of Biology, Guangdong Medical College, Zhanjiang, Guangdong 524023; \\ ${ }^{2}$ Modern Biochemistry Center, Guangdong Ocean University, Zhanjiang, Guangdong 524088, P.R. China
}

Received February 26, 2014; Accepted March 25, 2014

DOI: 10.3892/br.2014.267

\begin{abstract}
Naringenin, a flavonoid abundant in citrus fruits, such as grapefruits, has been reported to possess anti-inflammatory properties. The present study aimed to investigate the analgesic potential of naringenin in L5 spinal nerve ligation (SNL)-induced peripheral neuropathic pain and the underlying mechanisms associated with neuroinflammation. Different doses of naringenin or saline were administered intrathecally once daily for 11 consecutive days, from 3 days prior to surgery to 7 days after surgery. Pain development was assessed 1 day prior to and 7-14 days after surgery in terms of mechanical withdrawal threshold and thermal withdrawal latency. Astrocytic and microglial activation and production of inflammatory mediators were determined on day 14 after surgery. The results demonstrated that naringenin dose-dependently attenuated the mechanical allodynia and thermal hyperalgesia induced by SNL. Furthermore, naringenin significantly inhibited SNL-induced activation of glial cells (astrocytes and microglia). Morover, the upregulated expression of inflammatory mediators in neuropathic pain was significantly inhibited by naringenin. Our findings suggested that repeated administration of naringenin may alleviate neuropathic pain, possibly through inhibiting neuroinflammation.
\end{abstract}

\section{Introduction}

Neuropathic pain, caused by lesions to the somatosensory nervous system, in either the peripheral or the central components, is a major cause of distress in patients. The currently available analgesic drugs used for the treatment of chronic pain are not particularly effective in such cases, in part due to our incomplete understanding of the mechanisms underlying the development and maintenance of neuropathic pain. Substantial evidence has established that neuroinflammation,

Correspondence to: Dr Yun-Tao Zhao, Modern Biochemistry Center, Guangdong Ocean University, East Huguangyan Road, Zhanjiang, Guangdong 524088, P.R. China

E-mail: yuntaozhao@163.com

Key words: naringenin, neuropathic pain, neuroinflammation involving the activation of glia and the release of inflammatory mediators, contributes to neuropathic pain (1-3). Following nerve injury, inflammatory mediators are released from primary afferent terminals into the spinal cord and glial cells (microglia and astrocytes) in the spinal dorsal horn are activated by several inflammatory molecules. Glial activation leads to pro-inflammatory responses with pathological effects, such as neuronal hyperexcitability, neurotoxicity and chronic inflammation, resulting in neuropathic pain (4).

Naringenin and its glycoside naringin are flavonoids abundant in grapefruit and other citrus fruits and juices (5). Naringin is metabolised to naringenin (4',5,7-trihydroxyflavanone), which readily crosses the blood brain barrier (6). Previous studies demonstrated that naringenin and naringin attenuate the release of lipopolysaccharide-induced pro-inflammatory mediators in vitro (7-9) and in vivo (10). It was suggested that naringenin and naringin exert anti-inflammatory and neuroprotective effects and may be of therapeutic value in various neurodegenerative diseases. Naringin was previously reported to improve long-term learning and memory ability in a mouse model of Alzheimer's disease (11). Furthermore, naringin was shown to reduce cognitive deficits in kainic acid-induced status epilepticus models (12) and 3-nitropropionic acid-induced Huntington's disease models (13). In addition, treatment with naringin was found to inhibit diabetes-induced neuropathic pain (14). However, the potential effects of naringenin on nerve injury-induced neuropathic pain have not been investigated. Using a rat model of neuropathic pain following L5 spinal nerve ligation (SNL), the present study was undertaken to investigate the role of naringenin administered intrathecally in pain behaviors, pro-inflammatory cytokine and chemokine production, as well as glial activation.

\section{Materials and methods}

Experimental animals. Male Sprague-Dawley rats, weighing 220-250 g, were housed in a temperature-controlled room in plastic cages with free access to food and water at $22-25^{\circ} \mathrm{C}$ on a 12-h light/dark cycle. All the experimental protocols described in this study were approved by the regulations stipulated by Guangdong Medical College Animal Care Committee, which follows the protocol outlined in the Guide for the Care and Use of Laboratory Animals published by the US National Institute of Health (NIH publication no. 85-23, revised in 1985). 
SNL neuropathic pain model. Peripheral nerve injury was produced by SNL according to a previously described method (15). Briefly, under chloral hydrate anesthesia (300 mg/kg, intraperitoneally; Sigma-Aldrich, St. Louis, MO, USA), the dorsal vertebral column was surgically exposed from L4 to L6. The paraspinal muscles were separated from the spinal processes at the L4-L6 level and the L5 transverse process was carefully removed. The right L5 spinal nerves were exposed and tightly ligated distal to the dorsal root ganglion using 6-0 silk thread. The same procedure was followed in the sham-operated group, without ligation of the L5 spinal nerve.

Drug administration. Under chloral hydrate anesthesia (300 $\mathrm{mg} / \mathrm{kg}$, intraperitoneally), the rats were implanted with a PE-10 intrathecal catheter (BD Biosciences, Bedford, MA, USA) in the lumbar enlargement (close to the L4-L5 segments) for intrathecal drug administration. Following a 7-day recovery, the catheter placement was verified by observing transient hind paw paralysis induced by intrathecal injection of lidocaine $(2 \%, 5 \mu \mathrm{l})$. Animals that failed to show any paralysis were excluded from the experiments. Naringenin (Sigma-Aldrich) was dissolved in 5\% dimethyl sulfoxide and then diluted with $0.9 \%$ (w/v) saline solution. The animals were divided into 5 groups for administration: the sham-vehicle group (10 $\mu 1$ normal saline was injected into sham-operated rats) and SNL rats receiving treatment with naringenin (50, 100 and $200 \mathrm{mg} / \mathrm{kg}$ ) or vehicle (10 $\mu 1$ normal saline) once daily, from 3 days prior to surgery to 7 days after the surgery ( $n=6$ rats per treatment group).

Mechanical and thermal sensitivity measurement. Mechanical allodynia was assessed using von Frey filaments (Stoelting, Kiel, WI, USA) by experimenters who were blinded to group assignment. The ipsilateral hind paw was pressed with one of a series of von Frey filaments with gradually increasing stiffness $(2,4,6,8,10,15$ and $20 \mathrm{~g})$ applied to the plantar surface for 5-6 seconds for each filament. A positive paw withdrawal response was recorded if the animal briskly lifted the hind paw. The interval between trials was $\geq 5 \mathrm{~min}$. For each trial, the same hind limb was stimulated 10 times by a single von Frey filament prior to stimulation by the next larger filament. The minimal value that resulted in $\geq 6$ responses to 10 stimulations was recorded. To assess thermal sensitivity, paw withdrawal latencies to a radiant heat stimulus were measured using the paw flick test apparatus (IITC Life Science, Woodland Hills, CA, USA). The thermal withdrawal latency was recorded as the threshold of thermal sensitivity. Each hind paw was tested 5 times at 5-min intervals.

Tissue harvest. Following completion of the behavioral tests on day 14 postoperatively, the rats were deeply anesthetized and then perfused intracardially with $250 \mathrm{ml}$ cold saline. The ipsilateral L4-L5 spinal cord tissue rostral to the injury site was removed, dissected while on cold ice, removed quickly and placed in liquid nitrogen for subsequent assays.

Quantitative reverse transcription-polymerase chain reaction ( $q R T-P C R)$. Glial fibrillary acidic protein (GFAP) and macrophage antigen-1 (Mac-1) were used as markers of astrocytic and microglial activation, respectively. The rats were deeply anesthetized with chloral hydrate $(300 \mathrm{mg} / \mathrm{kg})$ and perfused transcardially with phosphate-buffered saline (pH 7.4) followed by $4 \%$ paraformaldehyde. Total RNA from the L5 spinal segments was extracted with TRIzol (Gibco-BRL Life Technologies Inc., Grand Island, NY, USA). Complementary DNA (cDNA) was synthesized with Oligo(dT) 12-18 using SuperScript ${ }^{\circledR}$ III Reverse Transcriptase for qRT-PCR (Invitrogen Life Technologies, Carlsbad, CA, USA). The sense primer for GFAP was 5'-GCTGGAGCA AGACAAACATTC-3' and the antisense primer was 5'-CCC TACCCACTCCTACATCGT-3'. The sense primer for Mac-1 was 5'-TGGCTGCTACTACCGTCCCT-3' and the antisense primer was 5'-CAGGTTGGAGCCGAATAGGT-3'. The sense primer for GAPDH was 5'-CCCCCAATG TATCCGTTGTG-3' and the antisense primer was 5'-TAGCCCAGGATGCCCTTTAGT-3'. Equal amounts of RNA $(1 \mu \mathrm{g})$ were used to prepare cDNA using $\mathrm{SYBR}^{\circledR}$ Premix Ex Taq ${ }^{\mathrm{TM}}$ (Takara Bio, Inc., Tokyo, Japan) and analysed with a qRT-PCR detection system (Applied Biosystems, Foster City, CA, USA). All the values were normalized to GAPDH expression.

ELISA. The spinal production of tumor necrosis factor- $\alpha$ (TNF- $\alpha$ ), interleukin-1 $\beta$ (IL-1 $\beta$ ) and monocyte chemoattractant protein-1 (MCP-1) was quantified by ELISA kits for rats according to the manufacturer's instructions (BioSource International, Camarillo, CA, USA for IL-1 $\beta$; R\&D Systems, Minneapolis, MN, USA for TNF- $\alpha$; and BioLegend, San Diego, CA, USA for MCP-1). The values were expressed as $\mathrm{pg} / \mathrm{mg}$ protein.

Statistical analysis. The values are presented as means \pm standard error of the mean (SEM). For behavioral data, the comparisons were performed using repeated measures of analysis of variance (ANOVA). For other data, comparisons were performed using ANOVA followed by Bonferroni tests. Data were analyzed using SPSS 13.0 software (SPSS, Inc., Chicago, IL, USA). P<0.05 was considered to indicate a statistically significant difference.

\section{Results}

Naringenin dose-dependently attenuates SNL-induced mechanical allodynia and thermal hypersensitivity. To determine whether naringenin prevents the development of mechanical allodynia and thermal hypersensitivity, different doses of naringenin $(50,100$ and $200 \mathrm{mg} / \mathrm{kg})$ were administered intrathecally for 11 days, from 3 days prior to surgery to 7 days after surgery and behavioral tests were performed. We observed that the SNL injury resulted in prominent mechanical allodynia and thermal hypersensitivity, as shown in the SNL + Veh group. Repeated intrathecal administration of naringenin produced a dose-dependent reduction of SNL-induced mechanical hyperalgesia and thermal hypersensitivity, as shown in Fig. 1. Furthermore, the analgesic effects of $200 \mathrm{mg} / \mathrm{kg}$ naringenin were prominent.

Effects of naringenin on glial activation markers. We investigated GFAP and Mac-1 expression on day 14 after surgery 

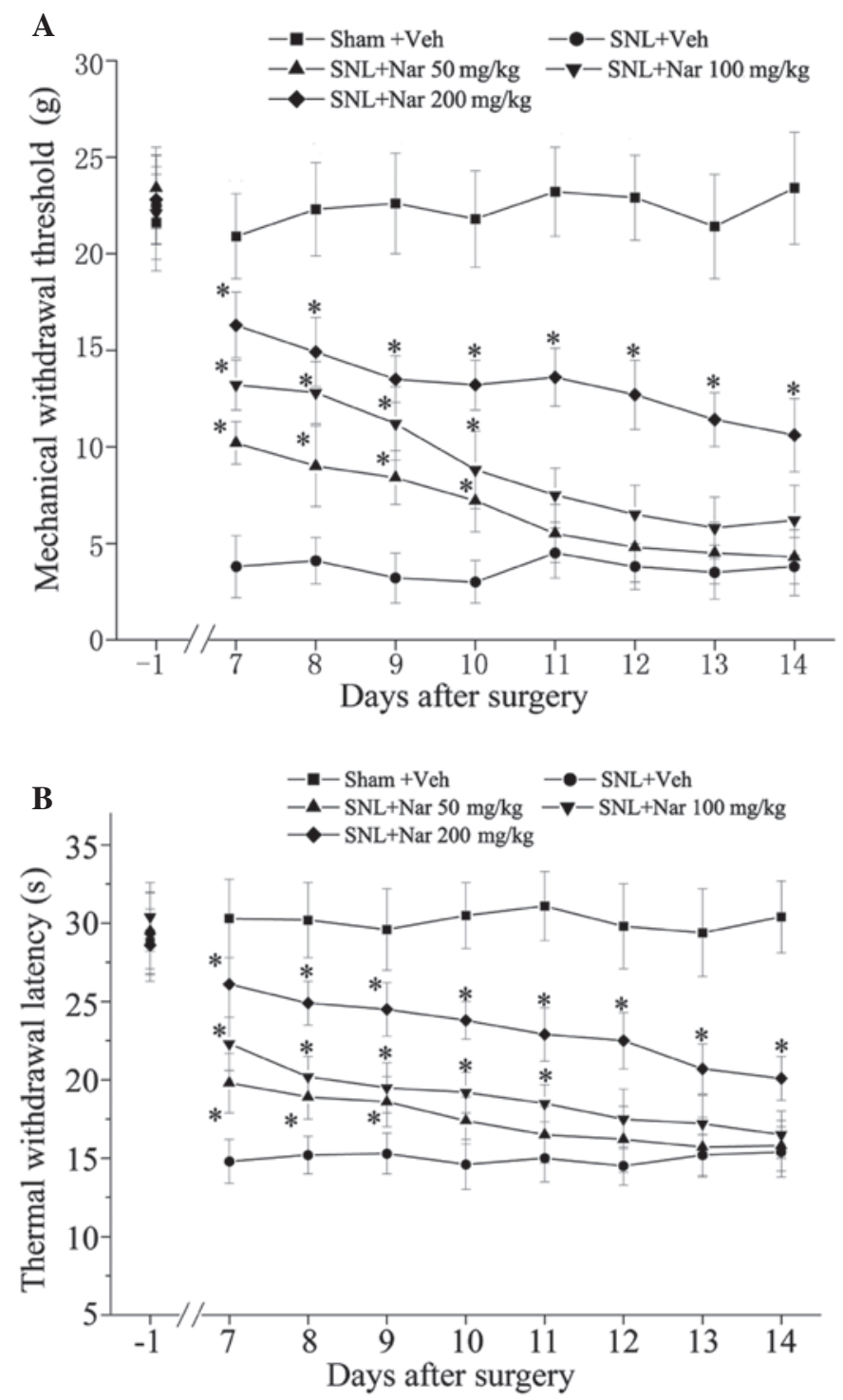

Figure 1. Repeated administration of naringenin (Nar) prevented spinal nerve ligation (SNL)-induced mechanical allodynia and thermal hyperalgesia. SNL injury resulted in (A) prominent mechanical allodynia and (B) thermal hyperalgesia on day 7 after surgery. The intrathecal administration of Nar (50, 100 or $200 \mathrm{mg} / \mathrm{kg}$, once per day, from 3 days prior to surgery to 7 days after surgery) significantly inhibited SNL-induced mechanical allodynia and thermal hyperalgesia in a dose-dependent manner. The values are expressed as means \pm standard error of the mean $(n=6)$. ${ }^{*} \mathrm{P}<0.05$, compared to the $\mathrm{SNL}+$ vehicle (Veh) group.
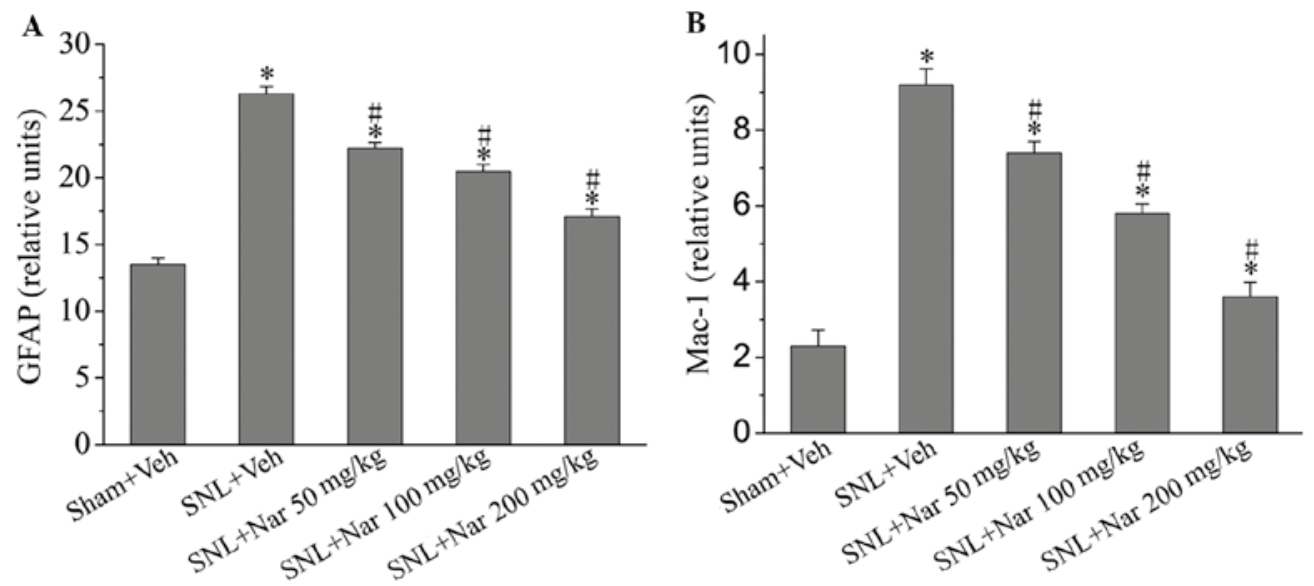

Figure 2. Naringenin (Nar) prevents spinal nerve ligation (SNL)-induced spinal glial activation. The expression of (A) astrocytic marker glial fibrillary acidic protein (GFAP) and (B) microglial marker macrophage antigen-1 (Mac-1) were measured by quantitative reverse trancription-polymerase chain reactions in the lumbar spinal cord of rats on day 14 after surgery. SNL increased GFAP and Mac-1 expression, whereas Nar dose-dependently downregulated the expression of these markers. The values are expressed as means \pm standard error of the mean $(\mathrm{n}=6)$. ${ }^{*} \mathrm{P}<0.05$ compared to the sham + vehicle (Veh) group. ${ }^{*} \mathrm{P}<0.05$ compared to the SNL + Veh group. 

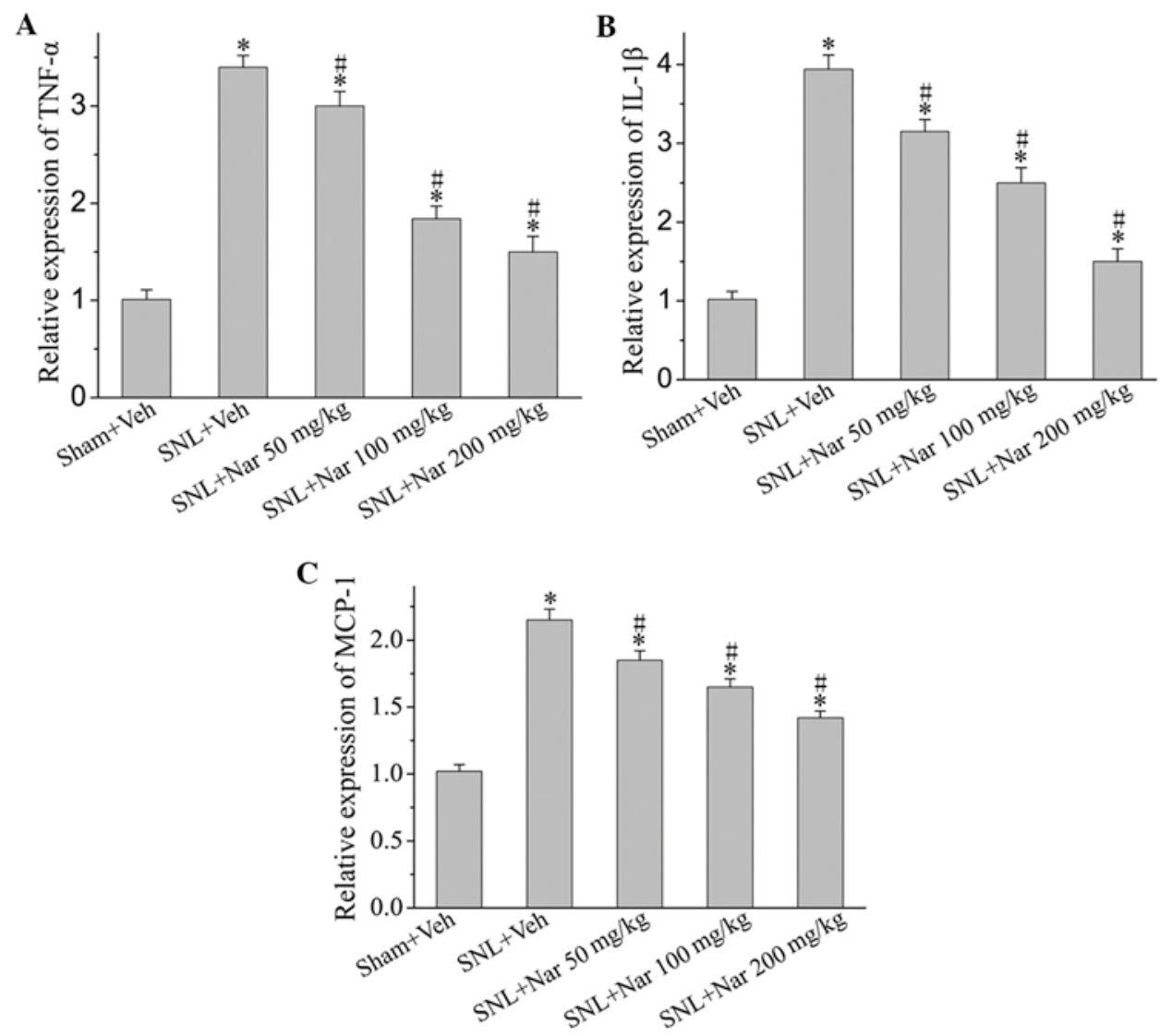

Figure 3. Effects of intrathecal naringenin (Nar) on pro-inflammatory cytokine levels. The protein levels of the pro-inflammatory cytokines (A) tumor necrosis factor- $\alpha$ (TNF- $\alpha$ ), (B) interleukin-1 $\beta$ (IL-1 $\beta$ ) and (C) monocyte chemoattractant protein-1 (MCP-1) were determined in the spinal dorsal horn of rats in different treatments groups using ELISA. Nar significantly prevented spinal nerve ligation (SNL)-induced increases in the levels of these inflammatory mediators. The values are expressed as means \pm standard error of the mean $(n=6)$. "P<0.05 compared to the sham + vehicle (Veh) group. ${ }^{*} \mathrm{P}<0.05$ compared to the $\mathrm{SNL}+\mathrm{Veh}$ group. The levels of the pro-inflammatory cytokines are expressed as relative fold-changes compared to the sham + Veh group.

in various groups to identify whether the analgesic effects of naringenin were accompanied by inhibition of glial activation. Compared to the sham group, the expression of GFAP and Mac-1 was significantly increased in the SNL + Veh group. Following treatment with naringenin, glial GFAP and Mac-1 expression levels were significantly decreased compared to those in the SNL + Veh group, although they remained higher compared to those in the sham rats. In particular, $200 \mathrm{mg} / \mathrm{kg}$ naringenin exerted significant inhibitory effects (Fig 2).

Effects of naringenin on SNL-induced elevated pro-inflammatory cytokine and chemokine expression. SNL-induced neuropathic pain was associated with a marked increase in the protein levels of pro-inflammatory cytokines and chemokines, such as TNF- $\alpha$, IL-1 $\beta$ and MCP-1. However, intrathecal naringenin administration achieved a significant decrease in the release of these pro-inflammatory cytokines and chemokines in a dose-dependent manner (Fig 3).

\section{Discussion}

In the present study, we investigated the analgesic effect of naringenin on the development of neuropathic pain and the possible underlying mechanisms. Our data demonstrated that repeated intrathecal administration of naringenin was able to dose-dependently attenuate mechanical allodynia and thermal hyperalgesia induced by SNL. Moreover, naringenin significantly inhibited peripheral neuropathy-induced spinal astrocytic and microglial activation. Furthermore, the synthesis of pro-inflammatory mediators in the spinal cord of neuropathic rats was suppressed by naringenin. These results suggested that naringenin prevents the development of neuropathic pain, possibly through inhibition of neuroinflammation.

Neuroinflammation is crucial in the pathogenesis of neuropathic pain (2). In the present study, the astrocytes marked by GFAP and microglia marked by Mac-1 were highly activated following SNL, which was consistent with the findings of previous studies (16-18). However, the astrocyte and microglial activation was suppressed by naringenin and this suppressive effect appeared to be parallel to the effect of naringenin on mechanical allodynia and thermal hyperalgesia. The release of multiple pro-inflammatory mediators from the activated glia (19) leads to a long-term alteration of synaptic transmission in the central nervous system and plays a key role in the development of neuropathic pain (20). In this study, naringenin suppressed the increase in the spinal TNF- $\alpha$, IL- $1 \beta$ and MCP-1 levels in rats following SNL, which was consistent with the effects of naringenin on mechanical allodynia and thermal hyperalgesia.

Naringin and naringenin exert a potent anti-inflammatory effect and have been used successfully in the treatment of inflammatory diseases, such as Alzheimer's disease (11). In the present 
study, we observed that repeated administration of naringenin was able to alleviate neuropathic pain in a dose-dependent manner. Furthermore, the anti-allodynia effect of naringenin $(200 \mathrm{mg} / \mathrm{kg}$ ) remained significant 7 days after discontinuation of the treatment. These data suggest that intrathecal treatment with naringenin exerts a strong antinociceptive effect. Once this effect is achieved, it may last over a long period of time. This characteristic indicates that naringenin may be suitable for the long-term treatment of chronic pain. Moreover, naringenin penetrates the blood brain barrier easily. Therefore, naringenin may be a useful drug in the treatment of neuropathic pain.

\section{Acknowledgements}

This study was supported by grants from the Doctoral Program of Guangdong Medical College, China (no. XB1021) and the Breeding Project of the Education Department of Guangdong Province, China (no. LYM10084).

\section{References}

1. Jha MK, Jeon S and Suk K: Glia as a link between neuroinflammation and neuropathic pain. Immune Netw 12: 41-47, 2012.

2. Kiguchi N, Kobayashi Y and Kishioka S: Chemokines and cytokines in neuroinflammation leading to neuropathic pain. Curr Opin Pharmacol 12: 55-61, 2012.

3. Ren K and Dubner R: Interactions between the immune and nervous systems in pain. Nat Med 16: 1267-1276, 2010.

4. Milligan ED and Watkins LR: Pathological and protective roles of glia in chronic pain. Nat Rev Neurosci 10: 23-36, 2009.

5. Hare JT and Elliott DP: Grapefruit juice and potential drug interactions. Consult Pharm 18: 466-472, 2003.

6. Zbarsky V, Datla KP, Parkar S, Rai DK, Aruoma OI and Dexter DT: Neuroprotective properties of the natural phenolic antioxidants curcumin and naringenin but not quercetin and fisetin in a 6-OHDA model of Parkinson's disease. Free Radic Res 39: 1119-1125, 2005.

7. Kanno S, Shouji A, Tomizawa A, Hiura T, Osanai Y, Ujibe M, Obara Y, Nakahata N and Ishikawa M: Inhibitory effect of naringin on lipopolysaccharide (LPS)-induced endotoxin shock in mice and nitric oxide production in RAW 264.7 macrophages. Life Sci 78: 673-681, 2006
8. Bodet C, La VD, Epifano F and Grenier D: Naringenin has anti-inflammatory properties in macrophage and ex vivo human whole-blood models. J Periodontal Res 43: 400-407, 2008.

9. Park HY, Kim GY and Choi YH: Naringenin attenuates the release of pro-inflammatory mediators from lipopolysaccharide-stimulated BV2 microglia by inactivating nuclear factor- $\mathrm{\kappa} \mathrm{B}$ and inhibiting mitogen-activated protein kinases. Int $\mathrm{J}$ Mol Med 30: 204-210, 2012.

10. Shiratori K, Ohgami K, Ilieva I, Jin XH, Yoshida K, Kase S and Ohno $\mathrm{S}$ : The effects of naringin and naringenin on endotoxin-induced uveitis in rats. J Ocul Pharmacol Ther 21: 298-304, 2005

11. Wang D, Gao K, Li X, Shen X, Zhang X, Ma C, Qin C and Zhang L: Long-term naringin consumption reverses a glucose uptake defect and improves cognitive deficits in a mouse model of Alzheimer's disease. Pharmacol Biochem Behav 102: 13-20, 2012.

12. Golechha M, Chaudhry U, Bhatia J, Saluja D and Arya DS: Naringin protects against kainic acid-induced status epilepticus in rats: evidence for an antioxidant, anti-inflammatory and neuroprotective intervention. Biol Pharm Bull 34: 360-365, 2011.

13. Kumar P and Kumar A: Protective effect of hesperidin and naringin against 3-nitropropionic acid induced Huntington's like symptoms in rats: possible role of nitric oxide. Behav Brain Res 206: 38-46, 2010.

14. Kandhare AD, Raygude KS, Ghosh P, Ghule AE and Bodhankar SL: Neuroprotective effect of naringin by modulation of endogenous biomarkers in streptozotocin induced painful diabetic neuropathy. Fitoterapia 83: 650-659, 2012.

15. Kim SH and Chung JM: An experimental model for peripheral neuropathy produced by segmental spinal nerve ligation in the rat. Pain 50: 355-363, 1992.

16. Feng X, Zhang F, Dong R, Li W, Liu J, Zhao X, Xue Q, Yu B and $\mathrm{Xu} \mathrm{J}$ : Intrathecal administration of clonidine attenuates spinal neuroimmune activation in a rat model of neuropathic pain with existing hyperalgesia. Eur J Pharmacol 614: 38-43, 2009.

17. Morgenweck J, Griggs RB, Donahue RR,Zadina JEand Taylor BK: PPARgamma activation blocks development and reduces established neuropathic pain in rats. Neuropharmacology 70: 236-246, 2013.

18. Chen Y, Willcockson HH and Valtschanoff JG: Influence of the vanilloid receptor TRPV1 on the activation of spinal cord glia in mouse models of pain. Exp Neurol 220: 383-390, 2009.

19. Reichling DB and Levine JD: Critical role of nociceptor plasticity in chronic pain. Trends Neurosci 32: 611-618, 2009.

20. Schomberg D and Olson JK: Immune responses of microglia in the spinal cord: contribution to pain states. Exp Neurol 234: 262-270, 2012. 